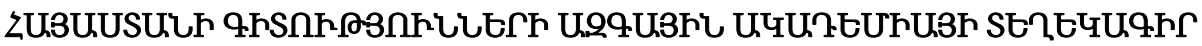 ИЗВЕСТИЯ НАЦИОНАЛЬНОЙ АКАДЕМИИ НАУК АРМЕНИИ
}

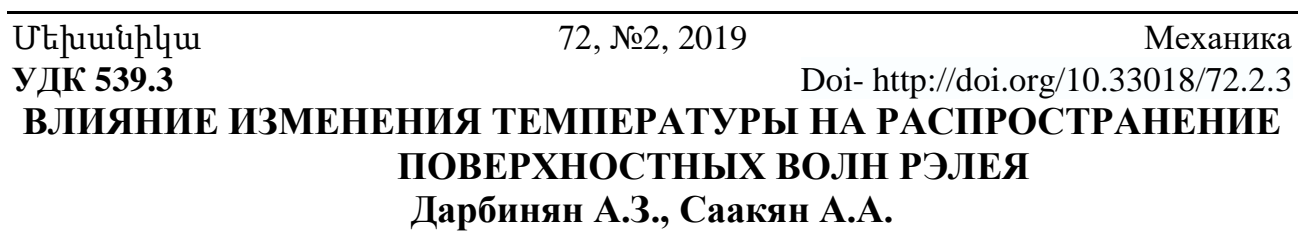

Ключевые слова: волна Рэлея, поперечная и продольная волны, теплопроводность, волновое число, частота, дисперсионное уравнение, условия затухания, гармоническая волна.

Darbinyan A.Z., Sahakyan A.A.

The effect of temperature on the propagation of Rayleigh surface waves.

Keywords: Rayleigh wave, transverse and longitudinal wave, thermal conductivity, wave number, frequency, dispersion equation, damping conditions, harmonic wave.

An elastic semi-space with a free surface, which is in a plane deformed state, is considered and the possibility of the appearance of Rayleigh-type surface waves depending on the temperature changing is investigated. The dependence curves of the velocity of the surface wave on the parameter, which includes the elastic and temperature coefficients, as well as the wave number and temperature increment, are plotted.

Tupphijuif U...., Uuhhulyjui U.U.

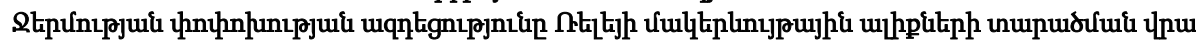

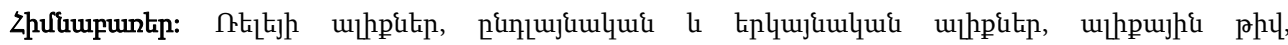

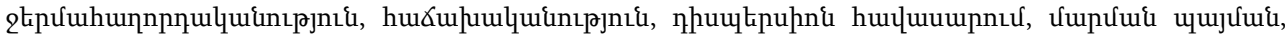
hupunfipl uihp.

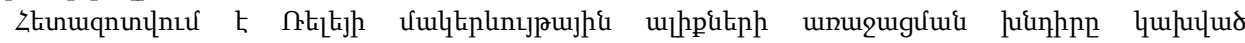

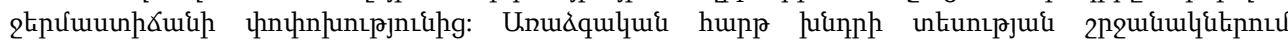

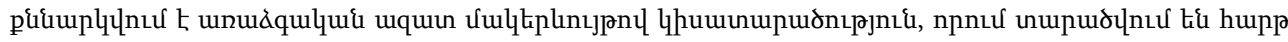

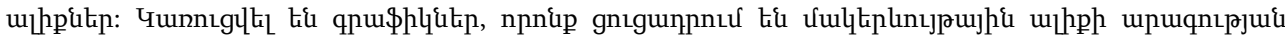

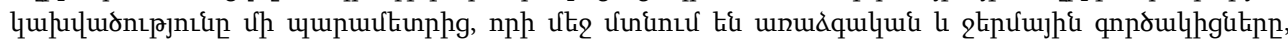

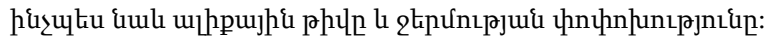

Рассматривается упругое полупространство со свободной поверхностью, находящееся в условиях плоского деформированного состояния. Построены графики зависимости скорости поверхностной волны от параметра, включающего в себя упругие и температурные коэффициенты, а также волновое число и перепад температуры. Исследуется возможность появления поверхностных волн типа Рэлея в зависимости от перепада температуры.

Введение. Упругие поверхностные волны хорошо изучены учёными и инженерами из-за их практической применимости к таким дисциплинам, как сейсмология, акустика, геофизика, материаловедение и другие [1-4]. Эти волны имеют особое значение в сейсмологии, так как, главным образом, они являются причиной разрушений во время землетрясения и, тем самым, наносят наибольший урон. И в настоящее время упругие поверхностные волны привлекают внимание инженеров-строителей, геологов и геофизиков, заинтересованных в сейсмологических приложениях.

Постановка задачи. Рассмотрим упругое полупространство $x_{1} \geq 0$ и предположим, что поверхностная волна распространяется в направлении оси $x_{2}$. Такого рода волна может возникнуть, если вызывающее её возмущение не зависит от пере- 
менной $x_{3}$ [5]. Поэтому $u_{3}=0$ и $\varepsilon_{33}=0, \varepsilon_{13}=0, \varepsilon_{32}=0$, то есть имеем плоскодеформированное состояние.

Предполагаем, что поверхность $x_{1}=0$ свободна от нормальных и касательных напряжений.

Уравнения движения в перемещениях с учётом температуры $T\left(x_{1}, x_{2}, t\right)$ и уравнения теплопроводности будут:

$$
\begin{aligned}
& (\lambda+\mu) \frac{\partial}{\partial x_{1}}\left(\frac{\partial u_{1}}{\partial x_{1}}+\frac{\partial u_{2}}{\partial x_{2}}\right)+\mu \Delta u_{1}-(3 \lambda+2 \mu) \alpha_{t} T=\rho \frac{\partial^{2} u_{1}}{\partial t^{2}} \\
& (\lambda+\mu) \frac{\partial}{\partial x_{2}}\left(\frac{\partial u_{1}}{\partial x_{1}}+\frac{\partial u_{2}}{\partial x_{2}}\right)+\mu \Delta u_{2}-(3 \lambda+2 \mu) \alpha_{t} T=\rho \frac{\partial^{2} u_{2}}{\partial t^{2}} \\
& \Delta T-\frac{1}{c_{3}^{2}} \frac{\partial^{2} T}{\partial t^{2}}=0
\end{aligned}
$$

где $u_{1}$ и $u_{2}$ - компоненты перемещения, $\lambda, \mu$ - постоянные Ламе, $\alpha_{t}-$ коэффициент линейного расширения, $\rho$ - плотность материала полуплоскости, $c_{3}=\sqrt{\lambda_{t} /\left(\tau_{r} c_{v}\right)}-$ скорость распространения тепла, $\lambda_{t}-$ коэффициент теплопроводности, $c_{v}$ - объёмная теплоёмкость, $\tau_{r}$ - время релаксации теплового потока, которое для металлов имеет величину $\tau_{r}=10^{-11}$ сек. [6].

Имеем граничные условия свободной поверхности:

$$
\begin{aligned}
& \sigma_{11}=\lambda\left(\frac{\partial u_{1}}{\partial x_{1}}+\frac{\partial u_{2}}{\partial x_{2}}\right)+2 \mu \frac{\partial u_{1}}{\partial x_{1}}-(3 \lambda+2 \mu) \alpha_{t}=0 \\
& \sigma_{12}=\mu\left(\frac{\partial u_{1}}{\partial x_{2}}+\frac{\partial u_{2}}{\partial x_{1}}\right)=0 .
\end{aligned}
$$

Относительно граничного условия для уравнения теплопроводности отметим, что оно получается путём упрощения уравнения теплопроводности, выписанного для очень тонкого слоя на поверхности полуплоскости. Предполагается, что коэффициент линейного расширения для слоя $\alpha_{t b}$ отличается от коэффициента $\alpha_{t}$ полуплоскости, а температура по толщине изменяется слабо. Тогда, из уравнения связанной теплопроводности $\frac{\partial^{2} T}{\partial x_{2}^{2}}+\frac{\partial^{2} T}{\partial x_{1}^{2}}+\frac{1}{c_{3}^{2}} \frac{\partial^{2} T}{\partial t^{2}}-\eta \frac{\partial^{2} u_{1}}{\partial x_{1} \partial t}=0 \quad$ [6,7] придём к следующему граничному условию:

$\frac{\partial T}{\partial x_{1}}-\eta \frac{\partial u_{1}}{\partial t}=0$ 
где $\eta=\frac{3 \lambda_{1}+2 \mu_{1}}{\lambda_{t 1}} \alpha_{t b} \delta T_{0}, \delta T_{0}-$ начальный перепад температуры [6].

\section{Решение задачи.}

Посредством скалярных потенциалов [5], $u_{1}=\frac{\partial \phi}{\partial x_{1}}+\frac{\partial \psi}{\partial x_{2}}, u_{2}=\frac{\partial \phi}{\partial x_{2}}-\frac{\partial \psi}{\partial x_{1}}$ уравнения (1) сводятся к уравнениям:

$\Delta \phi-\alpha_{0} T=\frac{1}{c_{1}^{2}} \frac{\partial^{2} \phi}{\partial t^{2}}, \quad \Delta \psi=\frac{1}{c_{2}^{2}} \frac{\partial^{2} \psi}{\partial t^{2}}, \quad \Delta T=\frac{1}{c_{3}^{2}} \frac{\partial^{2} \psi}{\partial t^{2}}$,

где $c_{1}^{2}=\frac{\lambda+2 \mu}{\rho}, \quad c_{2}^{2}=\frac{\mu}{\rho}, \quad \alpha_{0}=\frac{3 \lambda+2 \mu}{\lambda+2 \mu} \alpha_{t}$.

Граничные условия запишутся в виде

$2 \mu\left(\frac{\partial^{2} \phi}{\partial x_{1}^{2}}+\frac{\partial^{2} \psi}{\partial x_{1} \partial x_{2}}\right)+\lambda\left(\frac{\partial^{2} \phi}{\partial x_{1}^{2}}+\frac{\partial^{2} \phi}{\partial x_{2}^{2}}\right)-(3 \lambda+2 \mu) \alpha_{t} T\left(x_{1}, x_{2}, t\right)=0$,

$2 \frac{\partial^{2} \phi}{\partial x_{1} \partial x_{2}}+\frac{\partial^{2} \psi}{\partial x_{2}^{2}}-\frac{\partial^{2} \psi}{\partial x_{1}^{2}}=0 \quad$ при $x_{1}=0$

$\frac{\partial T}{\partial x_{1}}-\eta \frac{\partial}{\partial t}\left(\frac{\partial \phi}{\partial x_{1}}+\frac{\partial \psi}{\partial x_{2}}\right)=0$

Решение уравнений (2) будем искать в виде гармонических волн:

$\phi=\phi_{1}\left(x_{1}\right) e^{-i\left(\omega t-k x_{2}\right)}, \quad \psi=\psi_{1}\left(x_{1}\right) e^{-i\left(\omega t-k x_{2}\right)}, T=T_{1}\left(x_{1}\right) e^{-i\left(\omega t-k x_{2}\right)}$,

где $\omega$ - частота, а $k$-волновое число.

Необходимо обеспечить выполнение условий затухания на бесконечности в направлении $x_{1}$ :

$\lim _{x_{1} \rightarrow \infty} \phi_{1}\left(x_{1}\right)=0 ; \lim _{x_{1} \rightarrow \infty} \psi_{1}\left(x_{1}\right)=0 ; \lim _{x_{1} \rightarrow \infty} T_{1}\left(x_{1}\right)=0$.

Подставляя представления (6) в уравнения (4), получаем

$\phi_{1}^{\prime \prime}\left(x_{1}\right)-k^{2} v_{1}^{2} \phi_{1}\left(x_{1}\right)=\alpha_{0} T_{1}\left(x_{1}\right)$

$\psi_{1}^{\prime \prime}\left(x_{1}\right)-k^{2} v_{2}^{2} \psi_{1}\left(x_{1}\right)=0$

$T_{1}^{\prime \prime}\left(x_{1}\right)-k^{2} v_{3}^{2} T_{1}\left(x_{1}\right)=0$

Здесь введены обозначения:

$\xi=\frac{c^{2}}{c_{2}^{2}}, \quad \vartheta=\frac{\mu}{\lambda+2 \mu}=\frac{c_{2}^{2}}{c_{1}^{2}}, \quad \vartheta_{1}=\frac{c_{2}^{2}}{c_{3}^{2}}, \quad v_{1}^{2}=1-\vartheta \xi$,

$v_{2}^{2}=1-\xi, \quad v_{3}^{2}=1-\vartheta_{1} \xi, \quad c=\omega / k$.

Очевидно, что для обеспечения условий затухания (7) необходимо выполнение условия 
$\operatorname{Re} \sqrt{1-\xi}>0$.

Общее решение уравнений (8), удовлетворяющее условиям затухания (7), будет:

$$
\begin{aligned}
& \phi_{1}\left(x_{1}\right)=A e^{-k v_{1} x_{1}}+\frac{\alpha_{0}}{k^{2}\left(v_{3}^{2}-v_{1}^{2}\right)} B e^{-k v_{3} x_{1}}, \\
& \psi_{1}\left(x_{1}\right)=C e^{-k v_{2} x_{1}}, \quad T_{1}\left(x_{1}\right)=B e^{-k x_{1} v_{3}} .
\end{aligned}
$$

Удовлетворяя граничным условиям (2) и (3), получим систему однородных уравнений относительно коэффициентов $A, B, C$ :

$$
\begin{aligned}
& (2-\xi) A+\frac{\alpha_{0}}{k^{2}} \frac{2-\xi}{\xi\left(\vartheta-\vartheta_{1}\right)} B-2 i \sqrt{1-\xi} C=0 \\
& -2 i \sqrt{1-\vartheta \xi} A+\frac{2 i \alpha_{0} \sqrt{1-\vartheta_{1} \xi}}{k^{2}\left(\vartheta_{1}-\vartheta\right) \xi} B-(2-\xi) C=0 \\
& i \eta \omega \sqrt{1-\vartheta \xi} A+\sqrt{1-\vartheta_{1} \xi}\left(1+\frac{i \eta \alpha_{0} \omega}{k^{2} \xi\left(\vartheta-\vartheta_{1}\right)}\right) B+\eta \omega C=0 .
\end{aligned}
$$

Для существования ненулевого решения однородных линейных уравнений (12) необходимо равенство нулю главного детерминанта. Это условие приводит к следующему дисперсионному уравнению относительно $\xi$ :

$$
\begin{aligned}
& \sqrt{1-\vartheta_{1} \xi}\left[4 \sqrt{(1-\xi)(1-\vartheta \xi)}-(2-\xi)^{2}\right]+i \frac{\eta_{1}(2-\xi) \sqrt{\xi}}{\vartheta-\vartheta_{1}}\left(\sqrt{1-\vartheta_{1} \xi}-\sqrt{1-\vartheta \xi}\right)=0 \\
& \text { где } \eta_{1}=\frac{c_{2}\left(3 \lambda_{1}+2 \mu_{1}\right) \alpha_{t b}}{\lambda_{t 1}} \frac{(3 \lambda+2 \mu) \alpha_{t}}{(\lambda+2 \mu) k} \delta T_{0} .
\end{aligned}
$$

Действительная часть полученного уравнения в качестве множителя содержит известное уравнение Рэлея [1,5].

Выделив нулевой корень, будем иметь:

$$
\sqrt{1-\vartheta_{1} \xi}\left[\xi+4 \sqrt{(1-\xi)} \frac{\vartheta-1}{\sqrt{1-\xi}+\sqrt{1-\vartheta \xi}}\right]+i \frac{\eta_{1}(2-\xi)}{\left(\vartheta-\vartheta_{1}\right) \sqrt{\xi}}\left(\sqrt{1-\vartheta_{1} \xi}-\sqrt{1-\vartheta \xi}\right)=0
$$

Очевидно, что при наличии температурного поля, обусловленного тепловым потоком, распространяющимся с конечной скоростью, существование поверхностной волны будет зависеть от параметра $\eta_{1}$ во втором слагаемом уравнения (13).

Расмотрим частные случаи.

1. Пусть коэффициент линейного расширения полуплоскости $\alpha_{t}$ равен нулю, а, следовательно, и $\eta_{1}=0$. Тогда, очевидно, напряжённо-деформированное состояние полуплоскости не будет зависеть от изменения температуры и дисперсионное уравнение (13) перейдёт в известное уравнение Рэлея: 
$\sqrt{1-\vartheta_{1} \xi}\left[4 \sqrt{(1-\xi)(1-\vartheta \xi)}-(2-\xi)^{2}\right]=0$.

2. На свободной поверхности заданы условия Навье:

$\sigma_{11}=\lambda\left(\frac{\partial u_{1}}{\partial x_{1}}+\frac{\partial u_{2}}{\partial x_{2}}\right)+2 \mu \frac{\partial u_{1}}{\partial x_{1}}-(3 \lambda+2 \mu) \alpha_{t}=0$

$u_{2}=0, \frac{\partial T}{\partial x_{1}}-\eta \frac{\partial u_{1}}{\partial t}=0$

В этом случае дисперсионное уравнение будет

$\left.\sqrt{1-\xi}\left[\xi \sqrt{\left(1-\vartheta_{1} \xi\right.}\right)-i \frac{\eta_{1} \sqrt{\xi}}{\vartheta-\vartheta_{1}}\left(\sqrt{1-\vartheta \xi}-\sqrt{1-\vartheta_{1} \xi}\right)\right]=0$

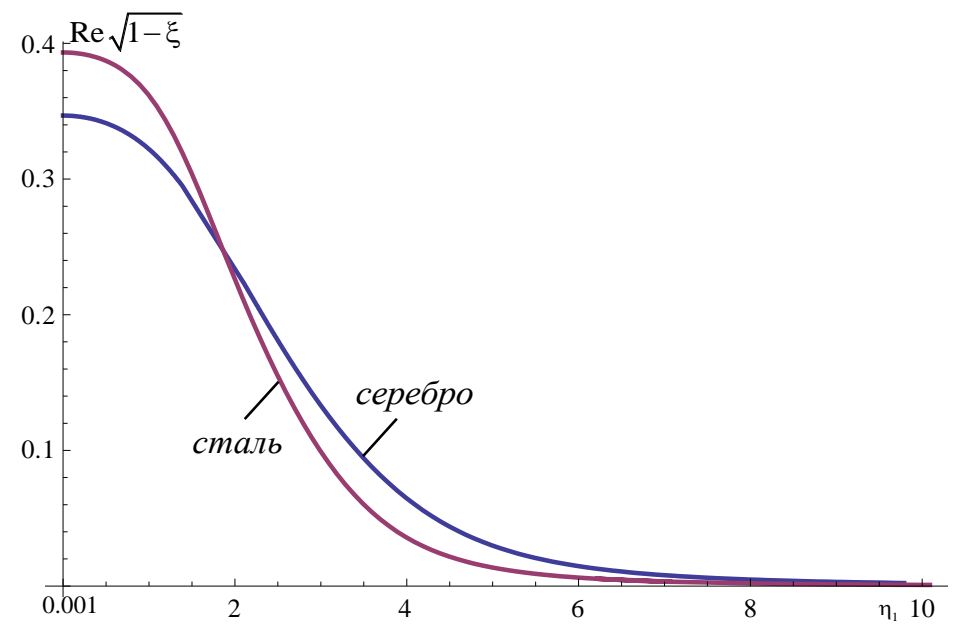

Фиг.1. Зависимость показателя убывания амплитуды поверхностной волны от $\eta_{1}$ в общем случае (формула (13)).

Расчёты показывают, что полученное дисперсионное уравнение имеет только два корня: $\xi=0$ и $\xi=1$, ни один из которых не допускает волнового процесса. Таким образом, при задании на границе полуплоскости условий Навье распространение поверхностной волны невозможно.

На фиг. 1 представлена зависимость показателя убывания амплитуды поверхностной волны от параметра $\eta_{1}$, характеризующего влияние изменения температуры, когда корень $\xi$ определяется из уравнения (13).

Выявлен диапазон появления поверхностных волн в зависимости от температуры для стали и серебра. Расчёты показывают, что при значении $\eta_{1}>1.226$ для стали и $\eta_{1}>1.34$ для серебра существует дополнительная поверхностная волна, скорость 
которой лежит между скоростями продольной и поперечной волн, подобный результат при наличии импеданса в граничных условиях получен и в работе [8].

Заключение. Показано, что наличие изменяющегося температурного поля приводит к расширению зоны локализации поверхностной волны, распространяя её влияние в глубь полупространства.

Авторы выражают благодарность профессору М.В.Белубекяну за постановку задачи и ценные указания при её решении.

\section{ЛИТЕРАТУРА}

1. Rayleigh L. On waves propagating along the plane surface of an elastic solid. Proc. R. Soc. London. A17 (1885) 4-11.

2. Achenbach J.D. Wave Propagation in Elastic Solids. North- Holland, Amsterdam, 1973, 465p.

3. Harris J.G. Linear Elastic Waves, Cambridge, New-York: 2001, 162p.

4. X.-F. Li On approximate analytic expressions for the velocity of Rayleigh waves. Wave Motion 44 (2006) 120-127.

5. Новацкий В. Теория упругости. М.: «Мир», 1975. 872с.

6. Лыков А.В. Тепломассобмен. М.: Энергия, 1971. 309c.

7. Белубекян М.В. Поверхностные волны в упругих средах. Проблемы динамики взаимодействия деформируемых сред. Ереван -1997. С.79-96.

8. Eduardo Goday, Mario Duran, Jean-Claude Nedelec. On the existence of surface waves in on elastic half-space with impedance boundary conditions. Wave Motion 49(2012)585-594.

\section{Сведения об авторах:}

Дарбинян Артавазд Завенович - к.ф.м.н., с.н.с. Института механики НАН Армении. E-mail: darbinyan_1954@mail.ru,

Саакян Арег Аветикович - к.ф.м.н., н.с. Института механики НАН Армении

E-mail: $\underline{\operatorname{areg} 1992 @ \text { gmail.com }}$

Поступила в редакцию 22. 02. 2019 г. 